\title{
Chemoembolization for Hepatocellular Carcinoma Supplied by a Lumbar Artery
}

\author{
Han Myun Kim • Hyo-Cheol Kim • Sungmin Woo • \\ Kyu Ri Son · Seong Whi Cho · Jin Wook Chung
}

Received: 2 March 2014/ Accepted: 3 April 2014/Published online: 22 May 2014

(C) Springer Science+Business Media New York and the Cardiovascular and Interventional Radiological Society of Europe (CIRSE) 2014

\begin{abstract}
Purpose To describe the radiologic findings and imaging response of hepatocellular carcinoma (HCC) supplied by the lumbar artery.

Methods Between April 2004 and December 2012, we encountered HCC supplied by a lumbar artery in 21 patients. Two investigators retrospectively reviewed clinical and radiological findings of HCC supplied by the
\end{abstract}

\section{H. M. Kim}

Department of Radiology, Kangnam Sacred Heart Hospital, Hallym University College of Medicine, Seoul, Korea

e-mail: seoul49@naver.com

H. M. Kim · S. W. Cho

Department of Radiology, Kangwon National University Hospital, Kangwon National University College of Medicine, Chuncheon, Korea

S. W. Cho

e-mail: chosw@kangwon.ac.kr

H.-C. Kim $(\bowtie) \cdot$ S. Woo $\cdot$ J. W. Chung

Department of Radiology, Seoul National University College of Medicine, Institute of Radiation Medicine, Seoul National

University Medical Research Center, and Clinical Research

Institute, Seoul National University Hospital, \# 101 Daehak-ro, Jongno-gu, Seoul 110-744, Korea

e-mail: angiointervention@gmail.com

S. Woo

e-mail: j_crew7@hotmail.com

J. W. Chung

e-mail: chungjw@snu.ac.kr

\section{K. R. Son}

Department of Radiology, Korea University Medical Center, Korea University College of Medicine, Seoul, Korea

e-mail:kyurad@gmail.com lumbar artery using computed tomography (CT) scans and digital subtraction angiograms.

Results Patients had received 1-27 sessions of previous chemoembolization procedures (mean 7.7 sessions, median 4 sessions). Mean tumor size was $5.3 \mathrm{~cm}$. The locations of HCC supplied by lumbar artery were the bare area $(n=14$, $67 \%)$ and segment VI $(n=7,33 \%)$. Tumor-feeding arteries arose from the main lumbar artery $(n=7)$, proximal anterior division $(n=4)$, and distal anterior division $(n=14)$. In 20 patients, selective chemoembolization through the tumor-feeding arteries of the lumbar artery was achieved. In 1 patient, nonselective embolization at the main lumbar artery was performed. There was no complication such as skin necrosis or paralysis. On the first follow-up enhanced CT scan, target tumors fed by the lumbar artery showed complete response $(n=6)$, partial response $(n=4)$, stable disease $(n=3)$, and progressive disease $(n=8)$, but overall tumor response was partial response $(n=1)$ and progressive disease $(n=20)$.

Conclusion When HCC is located in the inferior tip or bare area of the liver, a lumbar artery may supply the tumor. Although selective chemoembolization via the tumor-feeding vessel of the lumbar artery can be achieved in most cases, overall tumor response is commonly unfavorable.

Keywords Interventional Oncology ·

Chemoembolization/Chemoembolisation .

Liver/Hepatic

\section{Introduction}

Chemoembolization is a widely accepted treatment option in patients with unresectable hepatocellular carcinoma (HCC) $[1,2]$. Repeated chemoembolization is frequently 
undertaken to control the tumor, and development of extrahepatic collateral arteries supplying the tumor are common phenomena in daily clinical practice. Thus, the extrahepatic collateral arteries including inferior phrenic artery, intercostal artery, internal mammary artery, omental branch, renal artery, adrenal artery, and superior mesenteric artery should be investigated with consideration of the tumor location [3-7]. Among these, a right lumbar artery can infrequently supply HCC that is invading the abdominal wall or abuts the posteroinferior diaphragm [3]. Miyayama et al. [8] described 11 patients who received chemoembolization via the lumbar artery supplying HCC located in the bare area of the liver. During a period of 9 years, we treated 21 patients with HCC by chemoembolization via the lumbar artery in our institution. The purpose of this study was to evaluate the radiologic findings and imaging response of chemoembolization for the HCC supplied by the lumbar artery.

\section{Materials and Methods}

\section{Patients}

Since 1998, chemoembolization data were registered in an electronic database (Microsoft Access; Microsoft, Redmond, WA) immediately after the procedures. All radiological data were retrieved from these prospectively collected HCC database, and clinical information were reviewed retrospectively. Between January 2004 to December 2012, a total of 22,954 chemoembolization procedures were performed in 5540 patients with HCC in our institution. Among them, 22 patients $(0.4 \%)$ with HCC supplied by the lumbar artery were treated with chemoembolization. One patient was excluded from this retrospective study as a result of lack of follow-up enhanced computed tomographic (CT) scan. Thus, 21 patients were included in this study. All patients were men with ages ranging from 23 to 78 years (mean, 59.7 years). Liver cirrhosis was associated with hepatitis B in 17 patients and hepatitis $\mathrm{C}$ in 3 patients, and the other one was related to alcohol. Seventeen patients were Child-Pugh class A, and 4 patients were Child-Pugh class B. Our institutional review board approved this study; patient informed consent was waived for this retrospective study.

Methods of Chemoembolization and Follow-up

In all patients, enhanced CT scan was performed within 30 days before chemoembolization with various multidetector-row CT scanners. Celiac angiography was performed using a 5F Rosch hepatic catheter (Cook, Bloomington, IN), and selective angiography using a

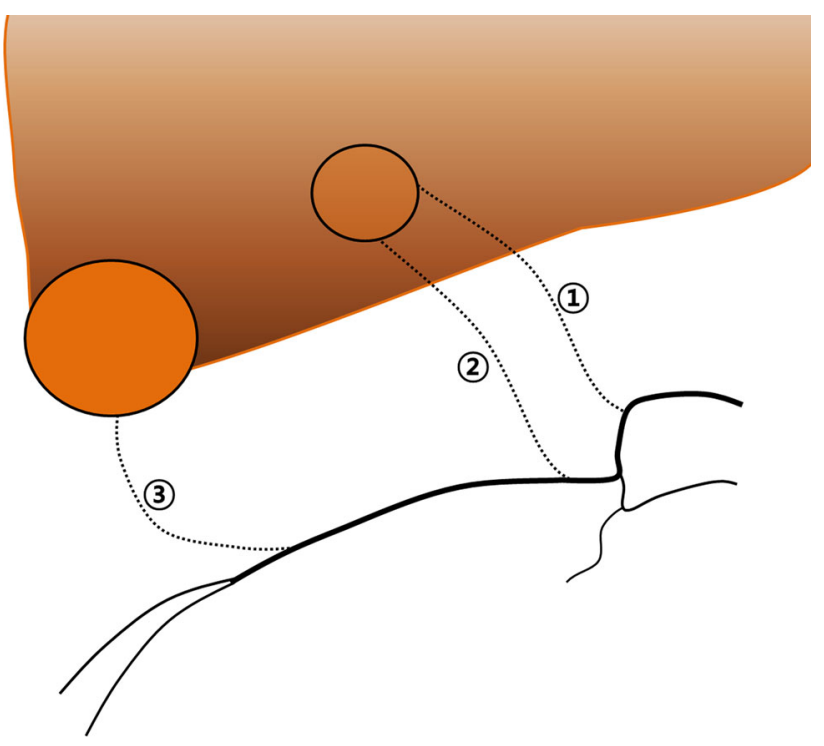

Fig. 1 Diagram of lumbar artery. 1 tumor-feeding artery arising from main lumbar artery, 2 tumor-feeding artery arising from proximal anterior division, 3 tumor-feeding artery arising from distal anterior division

microcatheter with a 2.0F tip (Progreat; Terumo, Tokyo, Japan) was performed for the hepatic artery or extrahepatic collateral vessels. Chemoembolization was performed by injection of mixture of iodized oil (Lipiodol; Andre Gurbet, Aulnay-sous-Bois, France) and doxorubicin hydrochloride (Adriamycin RDF; Ildong Pharmaceutical, Seoul, Korea) followed by additional embolization using gelatin sponge particles (Gelfoam; Upjohn, Kalamazoo, MI, or Cutanplast; MasciaBrunelli, Milano, Italy) or polyvinyl alcohol particles (Contour, Boston Scientific, Natick, Mass). When chemoembolization via the lumbar, intercostal, and internal mammary artery was performed, the right chest was uncovered and development of skin rash was evaluated during the procedure. Radicular pain or sensory alteration was not routinely assessed during or after the procedure unless patients complained of pain or sensory change.

Unenhanced CT scan was undertaken 2 weeks after chemoembolization to assess accumulation of iodized oil in the tumors that were observed on preprocedural enhanced CT scan. Enhanced CT scan was performed 1-3 weeks before a next scheduled chemoembolization session to evaluate residual or recurrent tumors. Routine interval between chemoembolization sessions is 3 months in our institute, but a repeated-treatment schedule was tailored to patient tolerance and tumor response.

Data Analysis

Two radiologists retrospectively reviewed the clinical, laboratory, and radiologic records of all patients in consensus. 
Table 1 Patient and tumor characteristics

\begin{tabular}{|c|c|}
\hline Characteristic & Value \\
\hline Age (year) & 59.7 \\
\hline Male sex & $100 \%$ \\
\hline \multicolumn{2}{|l|}{ Child-Pugh classification } \\
\hline A & 17 \\
\hline $\mathrm{B}$ & 4 \\
\hline \multicolumn{2}{|l|}{ Liver cirrhosis with: } \\
\hline HBsAg & 17 \\
\hline $\mathrm{HCVAb}$ & 3 \\
\hline Alcohol & 1 \\
\hline No. of previous chemoembolizations & $7.7(1-27)$ \\
\hline Tumor size $(\mathrm{cm})^{\mathrm{a}}$ & $5.3(1.6-11.2)$ \\
\hline \multicolumn{2}{|l|}{ Multiplicity of tumor } \\
\hline Single & 3 \\
\hline Multiple & 18 \\
\hline \multicolumn{2}{|l|}{ Tumor location } \\
\hline Segment VI & $7(33 \%)$ \\
\hline Segment VII & $14(67 \%)$ \\
\hline First lumbar artery & 19 \\
\hline Third lumbar artery & 2 \\
\hline \multicolumn{2}{|l|}{ Tumor-feeding artery } \\
\hline Single & 17 \\
\hline Multiple & 4 \\
\hline \multicolumn{2}{|l|}{ Origin of tumor-feeding artery ${ }^{\mathrm{b}}$} \\
\hline Main lumbar artery & 7 \\
\hline Proximal anterior division & 4 \\
\hline Distal anterior division & 14 \\
\hline
\end{tabular}

a In cases of multiple tumors, the size of tumors supplied by the lumbar artery was measured

b Three patients had 2 tumor-feeding arteries. One patient had several fine tumor-feeding arteries arising from the proximal and distal anterior division, and tumor-feeding arteries were counted as 2 in this patient

Tumors were classified as single nodule or multinodular. The size of tumors supplied by a lumbar artery was measured as the largest diameter on transverse CT scan. When the tumor was treated at the previous chemoembolization and iodized oil was partially accumulated in the tumor, tumor size was defined as the largest diameter of enhancing viable tumor. Tumor locations were classified into bare area or nonbare area. Bare area was defined as posterosuperior medial portion of right lobe that was in contact with the diaphragm [9, 10]. On CT scan, the bare area was outlined by the medial half of the right lobe and by the upper pole of the right kidney as the inferior level.

The origins of tumor-feeding branches were classified into main lumbar artery, proximal anterior division, and distal anterior division (Fig. 1). The proportion of blood supply by the lumbar artery was divided into exclusive
(100\% of tumor staining fed by the lumbar artery), dominant $(>50 \%)$, and supplementary $(<50 \%)$. We defined selective chemoembolization as selective catheterization of the tumor-feeding branch and delivery of treatment drugs.

The first follow-up enhanced CT scan was used to evaluate the response of the target tumor fed by the lumbar artery and overall tumor response using the modified Response Evaluation Criteria in Solid Tumors [11].

Tumor size was compared by $t$ test. A $P$ value of $<0.05$ was considered statistically significant. Statistical tests were performed by statistical software (SPSS 17.0; IBM, Armonk, NY).

\section{Results}

Radiological and clinical data are summarized in Tables 1 and 2. Patients had received 1-27 sessions of previous chemoembolization procedures (mean, 7.7 sessions; median, 4 sessions). The interval between initial chemoembolization and discovery of the lumbar artery ranged from 2.1 to 168.7 months (mean 35.8 months, median 11.2 months). All patients had a history of chemoembolization via extrahepatic collateral vessels during the previous procedures, including right inferior phrenic artery $(n=21)$, intercostal artery $(n=8)$, renal capsular artery $(n=6)$, adrenal artery $(n=4)$, omental branch $(n=3)$, left inferior phrenic artery $(n=3)$, right internal mammary artery $(n=2)$, right gastric artery $(n=2)$, renal artery $(n=2)$, left gastric artery $(n=1)$, and left internal mammary artery $(n=1)$.

Three patients had single nodular HCC, and 18 had multinodular tumors. Tumor size ranged from 1.6 to $11.2 \mathrm{~cm}$ (mean $5.3 \mathrm{~cm}$, median $5.2 \mathrm{~cm}$ ). Locations of HCC supplied by the lumbar artery were segment VII $(n=14,67 \%)$ and segment VI $(n=7,33 \%)$. Tumors in segment VII are located in the bare area, and tumors in segment VI had exophytic features in the right inferior tip of the liver. The tumors in segment VI $(6.8 \pm 3.1 \mathrm{~cm})$ were slightly larger than those in segment VII $(4.6 \pm 2.4 \mathrm{~cm})(P=0.092)$.

The levels of the lumbar artery to supply HCC were L1 $(n=19)$ and L3 $(n=2)$. Anterior spinal artery coming from the L1 was observed in three patients. The number of tumor-feeding branches was single $(n=17)$, double $(n=3)$, and several $(n=1)$. The tumor-feeding branches in 17 patients with a single feeder arose from the main lumbar artery $(n=5)$, the proximal anterior division $(n=2)$ (Fig. 2), and the distal anterior division $(n=10)$ (Fig. 3). The tumor-feeding branches in 4 patients with multiple feeders arose from the main lumbar artery and distal anterior division $(n=2)$ (Fig. 4) and the proximal and distal anterior division $(n=2)$. The relationship of tumor location and tumor-feeding arteries is summarized in Table 3. 


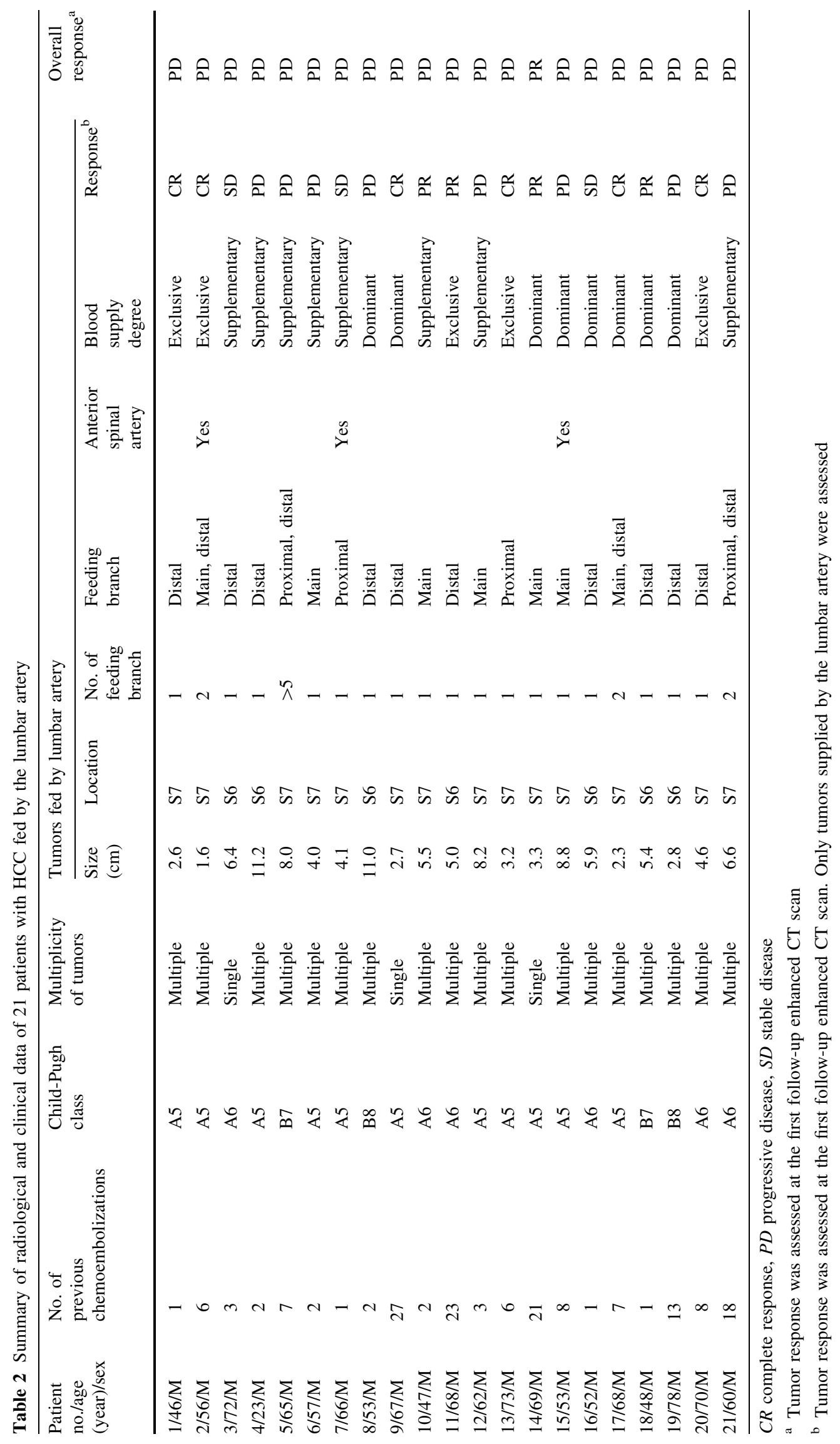



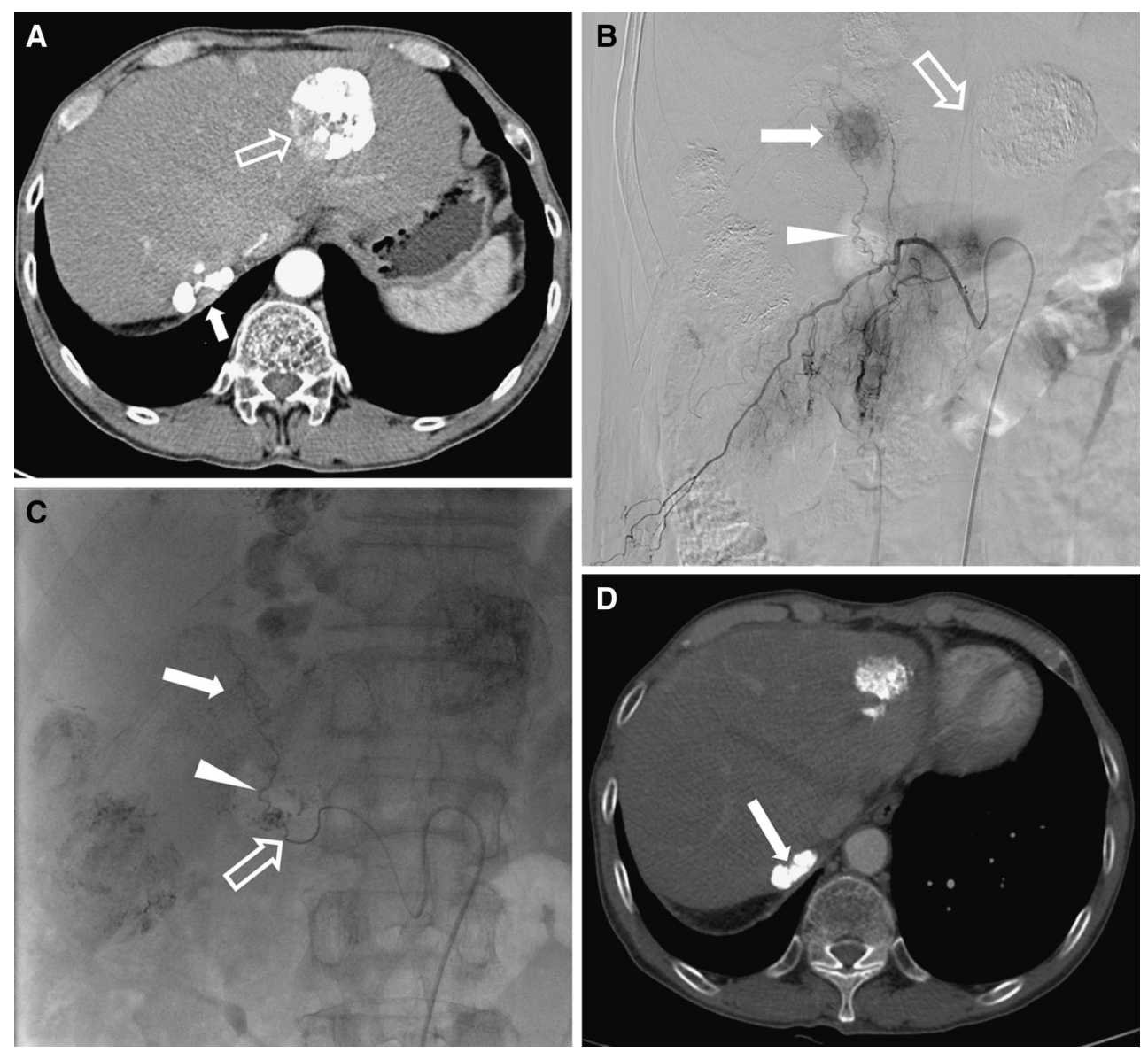

Fig. 2 Sixty-six-year-old man (patient 7) with HCC. A CT scan shows small enhancing nodule (arrow) in bare area. Note marginal recurrent tumor (open arrow) in left lobe. B Angiography of first lumbar artery at second session of chemoembolization shows tumor staining (arrow) supplied by tumor-feeding branch (arrowhead) arising from proximal anterior division of lumbar artery. Note anterior

The proportion of blood supplied by the lumbar artery was classified as exclusive $(n=5)$, dominant $(n=8)$, and supplementary $(n=8)$. In 11 patients, the tumors fed by the lumbar artery were also supplied by other extrahepatic collateral arteries, including intercostal artery $(n=7)$, right inferior phrenic artery $(n=4)$, renal capsular artery $(n=4)$, renal artery $(n=2)$, and adrenal artery $(n=1)$. Among 18 patients with multinodular tumor, 12 patients had 19 extrahepatic collateral arteries (7 right inferior phrenic arteries, 3 left inferior phrenic arteries, 3 adrenal arteries, 3 omental branches, 1 renal capsular artery, 1 intercostal artery, and 1 left gastric artery) supplying other tumors that were not fed by the lumbar artery.

In 20 patients, selective chemoembolization through the tumor-feeding arteries of the lumbar artery was achieved. In 1 patient with several tumor-feeding vessels, nonselective embolization at the main lumbar artery was performed using polyvinyl alcohol particles and gelatin sponge

spinal artery (open arrow) arising from posterior division of lumbar artery. C Image obtained during chemoembolization shows microcatheter tip (open arrow) in tumor-feeder branch. Note iodized oil emulsion in tumor-feeding branch (arrowhead) and tumor staining (arrow). D CT scan 5 weeks after chemoembolization shows dense accumulation of iodized oil (arrow) in tumor

particles. There were no complications such as skin necrosis or paralysis.

The mean interval between chemoembolization and the first follow-up enhanced CT scan was $57 \pm 17.5$ days (range 26-83 days, median 56 days). On the first follow-up enhanced CT scan, tumors fed by the lumbar artery showed complete response $(n=6)$, partial response $(n=4)$, stable disease $(n=3)$, and progressive disease $(n=8)$. Considering all tumors in the liver, 1 patient had partial response and 20 had progressive disease on the first follow-up CT scan.

\section{Discussion}

Lumbar artery can supply exophytic tumor in the inferior tip of the liver or small tumor in the bare area. Most patients had a history of previous chemoembolization of 

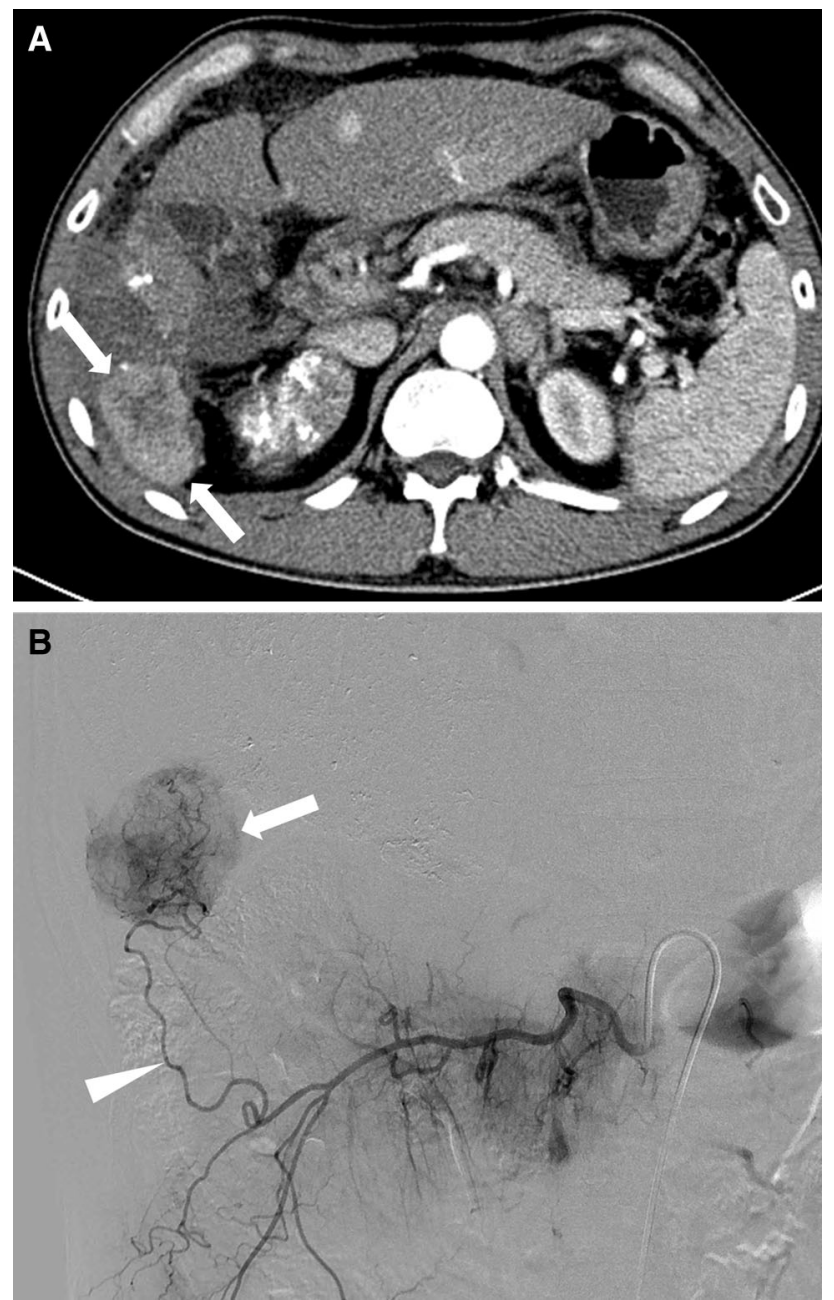

Fig. 3 Forty-eight-year-old man (patient 19) with HCC. A CT scan shows enhancing nodule (arrows) in inferior tip of liver. B Angiography of first lumbar artery at second session of chemoembolization shows tumor staining (arrow) supplied by tumor-feeding branch (arrowhead) arising from distal anterior division of lumbar artery

extrahepatic collateral vessels including right inferior phrenic artery. Tumor-feeding vessels may arise from the main lumbar artery as well as anterior branch of the lumbar artery. Selective chemoembolization via the tumor-feeding vessel can be achieved in most cases. Tumor response was commonly unfavorable because of concomitant other extrahepatic collateral arteries and the multiplicity of current tumors.

The lumbar arteries branch into an anterior and posterior division soon after they arise from the dorsolateral aspect of the aorta [12]. The posterior division consists of muscular branches and radicular branches that come off the anterior or posterior spinal arteries. The anterior division supplies back muscles in the lumbar area and may anastomose with subcostal, iliolumbar, deep circumflex iliac, and inferior epigastric arteries.

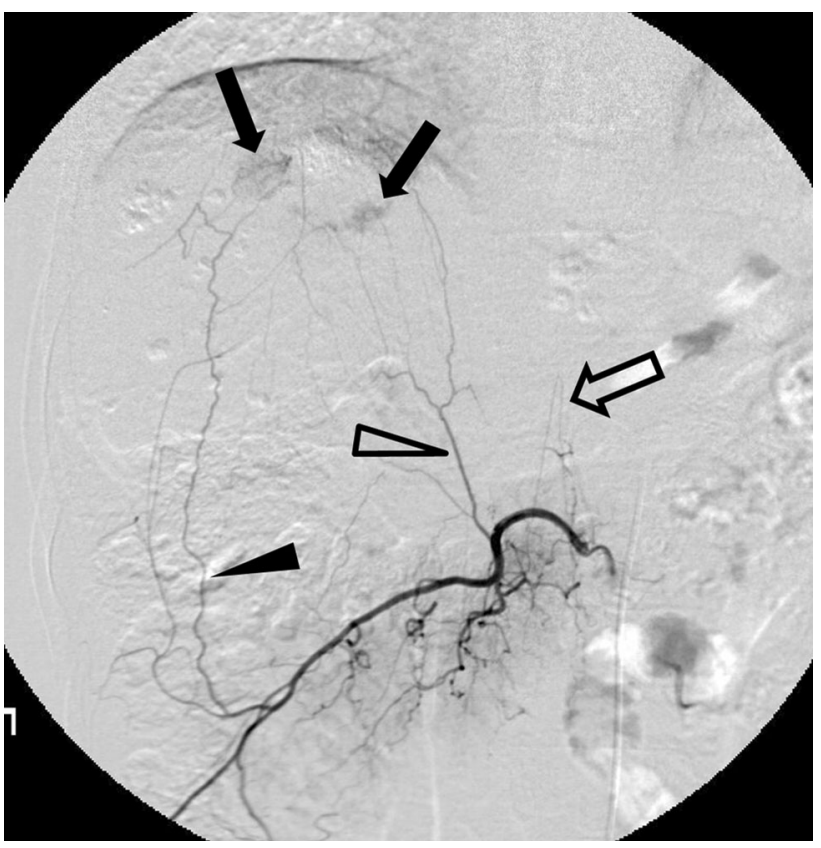

Fig. 4 Fifty-six-year-old man (patient 2) with HCC. Angiography of first lumbar artery at seventh session of chemoembolization shows tumor staining (arrows) supplied by tumor-feeding branch (arrowhead) arising from distal anterior division and by tumor-feeding branch (open arrowhead) arising from main lumbar artery. Anterior spinal artery (open arrow) arises from posterior division of lumbar artery

Table 3 Relationship of tumor location and tumor-feeding vessels

\begin{tabular}{lll}
\hline Origin of tumor-feeding vessel & $\begin{array}{l}\text { Tumor location } \\
\text { Bare area }\end{array}$ & Nonbare area \\
\hline Main lumbar artery & 5 & 0 \\
Proximal anterior division & 2 & 1 \\
Distal anterior division & 3 & 7 \\
Main lumbar artery/ & 2 & 0 \\
$\quad$ distal anterior division & & 0 \\
Proximal/distal anterior division & 2 &
\end{tabular}

In a previous study, $10(91 \%)$ of 11 tumors fed by the lumbar artery were located in the bare area of segment VII, and tumor-feeding arteries came from the main lumbar artery proximal to the bifurcation of anterior and posterior division in $8(73 \%)$ of 11 patients. In this study, 14 (67\%) of 21 tumors supplied by the lumbar artery were located in the bare area of segment VII, and tumor-feeding arteries arose from the main lumbar artery in 7 patients $(32 \%)$. We think that Miyayama et al. [8] were able to detect small tumors better which were located in the bare area and were supplied by the lumbar artery.

In patients with exophytic tumor in the inferior tip of the liver, it is easy to think about the possibility of the lumbar 
artery supplying the tumor. However, in patients with tumor in the bare area, we think that most radiologists will not try to perform angiography of the lumbar artery. Tumors in the bare area of segment VII are commonly supplied by the right inferior phrenic artery, and infrequently by the intercostal, adrenal, and renal capsular arteries $[3,13,14]$. In this study, the right inferior phrenic artery was embolized at the previous chemoembolization sessions in all 21 patients. When these collateral vessels have been embolized by previous procedures, the collateral supply from the lumbar artery subsequently develops. Thus, when small tumor was present in the bare area and was not able to be demonstrated on angiograms of hepatic, inferior phrenic, adrenal, and intercostal arteries, the lumbar artery should be interrogated.

There are unresolved issues concerning chemoembolization via the lumbar arteries. First, what is the best way to identify early the lumbar artery feeding tumors? We think that the most important thing is careful correlation between angiography and preprocedural CT. If there are missing tumors on angiography, we have to interrogate possible extrahepatic collateral arteries considering tumor location. After we retrospectively reviewed the previous CT scan and angiography, we concluded that we missed tumors fed by the lumbar artery at the previous chemoembolization sessions in 5 patients. In addition, it is also important to perform close and careful postchemoembolization imaging follow-up and to carefully review these images before the procedures. If we detect viable tumors untreated at the previous procedure, we should tune the procedural planning at the next session. Second, can $\mathrm{C}$-arm CT be helpful to detect tumors fed by the lumbar artery? It was reported that C-arm CT can improve detection of extrahepatic collateral arteries and can reduce the missing tumors [15], and we also agree with this opinion. Because the lumbar artery is the last artery that interventional radiologists suspect, however, we performed angiograms of several extrahepatic collateral arteries, including the inferior phrenic artery, intercostal artery, and renal capsular artery, before performing angiograms of the lumbar artery in most cases. Thus, C-arm CT of all these extrahepatic collateral arteries as well as the hepatic artery may increase the radiation exposure to the patients. Third, is aortography needed? We do not think that aortography is useful to detect the lumbar artery. Multidetector CT scan can frequently show the hypertrophied extrahepatic collateral arteries and their tumor-feeding branches. Thus, we can decide which extrahepatic collateral arteries should be interrogated before the procedures. We could not observe small tumorfeeding branches arising from the lumbar artery on CT scan, but it is also difficult to detect small tumor-feeding braches on aortography. Excessive use of contrast media is needed to detect small tumor-feeding branches by aortography, but it is still difficult to recognize small arteries on aortography. Fourth, can chemoembolization via the lumbar artery improve patients' survival? We think that chemoembolization via the lumbar artery does not improve survival in most patients because most patients have an advanced stage of disease, and patient survival did not depend on tumors fed by the lumbar artery. When primary tumors are well controlled by chemoembolization and small recurrent tumor is exclusively supplied by the lumbar artery, chemoembolization via the lumbar artery may affect patients' clinical outcome.

Spinal cord injury after chemoembolization is rare [16, 17], but it leads to a poor clinical outcome. Thus, interventional radiologists should pay attention to the anterior spinal artery arising from the intercostal or lumbar artery. The anterior spinal artery commonly arises from the intercostal artery, but the tumor-feeding branch almost always arises from the far distal portion of the intercostal artery [18]. However, the main lumbar artery commonly gives off tumor-feeding branch proximal to the bifurcation [8]. In this study, most tumor-feeding branches were able to be catheterized with a $2 \mathrm{~F}$ tip microcatheter, and there was no spinal cord injury. When the anterior spinal artery is evident on angiography, the tumor-feeding artery arises from the main lumbar artery, and selective catheterization cannot be achieved, an attempt to perform chemoembolization via the lumbar artery may cause serious complications.

There are several limitations to this study. First, this was a retrospective and nonrandomized observational study and dealt with narrow range of patients. Second, we did not use $\mathrm{C}$-arm CT in this study. C-arm CT can improve recognition of extrahepatic collateral vessels supplying the tumors [15]. Thus, we might have missed some lumbar arteries supplying tumors, or we might have discovered a lumbar artery supplying tumors after a delay of several months. Third, the timing of follow-up CT scan varied in this study, so tumor response at the first follow-up CT may be affected by the timing of CT scan.

In conclusion, when the HCC is located in the inferior tip or bare area of the liver, the lumbar artery may supply the tumor as an extrahepatic collateral pathway. Selective chemoembolization via the tumor-feeding vessel of the lumbar artery can be achieved in most cases.

Acknowledgments This study was supported by a Grant from the National R\&D Program for Cancer Control, Ministry of Health \& Welfare, Republic of Korea (1220040).

Conflict of interest Han Myun Kim, Hyo-Cheol Kim, Sungmin Woo, Kyu Ri Son, Seong Whi Cho, and Jin Wook Chung declare that they have no conflict of interest. 


\section{References}

1. Brown DB, Geschwind JF, Soulen MC et al (2009) Society of interventional radiology position statement on chemoembolization of hepatic malignancies. J Vasc Interv Radiol 20(7):317-323

2. Shin SW (2009) The current practice of transarterial chemoembolization for the treatment of hepatocellular carcinoma. Korean J Radiol 10:425-434

3. Kim HC, Chung JW, Lee W et al (2005) Recongnizing extrahepatic collateral vessels that supply hepatocellular carcinoma to avoid complications of transcatheter arterial chemoembolization. Radiographics 25(suppl):S25-S39

4. Kim HC, Chung JW, Choi SH et al (2007) Hepatocellular carcinoma with internal mammary artery supply: feasibility and efficacy of transarterial chemoembolization and factors affecting patient prognosis. J Vasc Interv Radiol 18:611-619

5. Kim HC, Chung JW, Kim WH et al (2010) Chemoembolization of the left inferior phrenic artery in patients with hepatocellular carcinoma: 9-year single-center experience. AJR Am J Roentgenol 194:1124-1130

6. Hur S, Kim HC, Chung JW et al (2011) Hepatocellular carcinomas smaller than $4 \mathrm{~cm}$ supplied by the intercostal artery: can we predict which intercostal artery supplies the tumor? Korean $\mathrm{J}$ Radiol 12:693-699

7. Choi JW, Kim HC, Chung JW et al (2012) Chemoembolization via branches from the splenic artery in patients with hepatocellular carcinoma. Cardiovasc Intervent Radiol 35:90-96

8. Miyayama S, Yamashiro M, Okuda M et al (2010) Hepatocellular carcinoma supplied by the right lumbar artery. Cardiovasc Intervent Radiol 33:53-60

9. Thornton FJ, Kandiah SS, Monkhouse WS, Lee MJ (2001) Helical CT evaluation of the perirenal space and its boundaries: a cadaveric study. Radiology 218:659-663
10. Miele V, Andreoli C, De Cicco ML et al (2002) Hemoretroperitoneum associated with liver bare area injuries: CT evaluation. Eur Radiol 12:765-769

11. Lencioni R, Llovet JM (2010) Modified RECIST (mRECIST) assessment for hepatocellular carcinoma. Semin Liver Dis 30:52-60

12. Johnsrude IS, Jackson DC, Dunnick NR (1987) A practical approach to angiography. Little Brown, Boston, pp 462-464

13. Miyayama S, Yamashiro M, Okuda M et al (2010) The March of extrahepatic collaterals: analysis of blood supply to hepatocellular carcinoma located in the bare area of the liver after chemoembolization. Cardiovasc Intervent Radiol 33:513-522

14. Ishikawa M, Yamagami T, Kakizawa H et al (2014) Transarterial therapy of hepatocellular carcinoma fed by the right renal capsular artery. J Vasc Interv Radiol 25:389-395

15. Tognolini A, Louie JD, Hwang GL et al (2010) Utility of C-arm $\mathrm{CT}$ in patients with hepatocellular carcinoma undergoing transhepatic arterial chemoembolization. J Vasc Interv Radiol 21: 339-347

16. Chung JW, Park JH, Han JK et al (1996) Hepatic tumors: predisposing factors for complications of transcatheter oily chemoembolization. Radiology 198:33-40

17. Kim JH, Yeon JE, Jong YK et al (2010) Spinal cord injury subsequent to transcatheter arterial chemoembolization in patients with hepatocellular carcinoma. Dig Liver Dis 42:67-70

18. Kim HC, Chung JW, Lee IJ et al (2011) Intercostal artery supplying hepatocellular carcinoma: demonstration of a tumor feeder by $\mathrm{C}$-arm CT and multidetector row CT. Cardiovasc Intervent Radiol 34:87-91 